\title{
Laringoplastía de inyección con ácido hialurónico, experiencia del Hospital Clínico de la Pontificia Universidad Católica de Chile
}

\section{Laryngoplasty injection with hyaluronic acid, Hospital Clinico de la Pontificia Universidad Catolica de Chile experience}

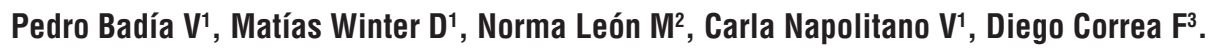

\begin{abstract}
RESUMEN
Introducción: El manejo inicial de la parálisis cordal unilateral incluye la observación en espera de la recuperación de la movilidad o la compensación, terapia fonoaudiológica o inyección de medialización temporal para llevar la cuerda a una posición más favorable para el cierre glótico.

Este procedimiento puede realizarse en pabellón, o vía percutánea en paciente despierto. Existen varias opciones, siendo el ácido hialurónico (Restylane®) uno de los más usados.

Objetivo: Revisar la experiencia pionera en el país en la inyección de medialización de cuerda vocal con ácido hialurónico en el manejo de la parálisis cordal unilateral.

Material y método: Estudio retrospectivo, que incluyó a 15 pacientes con insuficiencia glótica a los que se les realizó una inyección percutánea con ácido hialurónico. Se evaluó la calidad de la voz pre y posprocedimiento (VRQOL), tolerancia al procedimiento y complicaciones.

Resultados: El 100\% de los pacientes mostró cambios significativos en el VRQOL, con un cambio promedio pre y post de $37 \%$. El procedimiento fue bien tolerado en el 93\%. No se produjeron incidentes durante la inyección ni tampoco posteriores.

Conclusión: La inyección de medialización es un procedimiento bien tolerado, seguro y sencillo, que permite mejoras significativas en la calidad vocal. El ácido hialurónico es seguro y eficaz para la inyección de medialización.
\end{abstract}

Palabras clave: Parálisis unilateral cuerda vocal, inyección cuerda vocal, ácido hialurónico, laringoplastía de inyección.

\footnotetext{
Médico del Servicio de Otorrinolaringología. Pontificia Universidad Católica de Chile.

Fonoaudióloga del Servicio de Otorrinolaringología. Pontificia Universidad Católica de Chile.

3 Médico-Cirujano. Pontificia Universidad Católica de Chile.
}

Recibido el 30 de julio de 2015. Aceptado el 16 de agosto de 2015. 


\begin{abstract}
Introduction: Initial treatment options for unilateral vocal fold paralysis include observation for spontaneous return of function or compensation, voice therapy to stimulate recovery of motion or function, or temporary vocal fold injection medialization to improve glottic closure.

Temporary injection medialization can be performed under general anesthesia or in the awake-patient setting with local anesthesia. There are different options of temporary

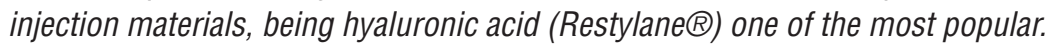

Aim: To review our pioneer experience in Chilewith the use of temporary vocal fold injection with hyaluronic acid in the initial management of unilateral vocal fold paralysis.

Material and method: Retrospective study involving 15 patients with glottic insufficiency, who underwent percutaneous vocal fold injection medialization with hyaluronic acid. Pre and post procedure quality of voice (VRQOL), tolerance to the procedure, and complications were evaluated.

Results: All patients showed significant changes in VRQOL, with a 37\% average improvement.The procedure was well tolerated in 93\%. No incidents occurred during injection orlater.

Conclusions: The temporary vocal fold injection medialization is a well-tolerated, safe and simple procedure that allows significant short-term improvements in vocal quality of patients. Hyaluronic acid is a safe and effective material for temporary vocal foldinjection medialization.

Key words: Unilateral vocal fold paralysis, vocal fold injection, hyaluronic acid, injection laryngoplasty.
\end{abstract}

\section{INTRODUCCIÓN}

La disfonía es un motivo de consulta común en otorrinolaringología, siendo la insuficiencia glótica secundaria a parálisis unilateral de cuerda vocal una de sus causas frecuentes. Tradicionalmente, el manejo inicial de estos pacientes ha consistido en la observación en espera de la recuperación espontánea de la movilidad cordal 0 de la compensación, o la terapia fonoaudiológica para favorecer la recuperación de la función'1.

En los últimos años se ha introducido la inyección de medialización temporal de la cuerda vocal como otra opción en el manejo inicial de esta patología, cuyo objetivo es disminuir temporalmente la insuficiencia glótica en espera de la recuperación de la movilidad o la compensación². Esta técnica fue introducida por Wilhelm Brunings ${ }^{3}$ en 1911 e implementada por Arnold en los años $60^{\circ}$. Posteriormente, en 1985 la técnica de abordaje transcervical fue introducida por Paul Ward ${ }^{4}$, lo que sumado al rápido avance de las tecnologías endoscópicas y al surgimiento de nuevos materiales de inyección, permitieron desarrollar nuevos abordajes percutáneos y mejorar la precisión en el sitio de inyección ${ }^{5}$.

La laringoplastía de inyección consiste en lograr, mediante la inyección de un agente reabsorbible, la medialización de la cuerda vocal inmóvil, dejándola en una posición más favorable para el cierre glótico ${ }^{6-7}$. Además puede mejorar la deglución en los pacientes que presentan este problema asociado a la parálisis cordal ${ }^{8}$.

Este procedimiento puede realizarse mediante laringoscopía directa bajo anestesia general en pabellón, o vía inyección percutánea en paciente despierto. Existen diversos materiales sintéticos y biológicos que se utilizan en la inyección de medialización de cuerda vocal ${ }^{8-9}$. Dentro de los materiales inyectables de larga duración se encuentran la hidroxiapatita de calcio (Radiesse Voice ${ }^{\circledR}$ ) y el polidimetilsiloxano (PDMS o silicona particulada). Dentro de los materiales temporales están el colágeno (Cymetra ${ }^{\circledR}$ ), carboximetilcelulosa (Radiesse 
Voice $\mathrm{Gel}^{\circledR}{ }^{\circledR}$, gelatina bovina (Gelfoam ${ }^{\circledR}$ ) y el ácido hialurónico (Restylane $\left.{ }^{\circledR}\right)$.

Últimamente se ha visto una preferencia por los materiales de inyección temporales, ya que se reabsorben en un lapso de tiempo de entre 3 y 6 meses, permitiendo el concepto de "inyección de prueba" y reversibilidad en caso de resultado no satisfactorio, pero con la posibilidad de inyecciones sucesivas en el tiempo en caso contrario. Además, existe evidencia de que los pacientes con parálisis unilateral de cuerda vocal que son sometidos precozmente a una inyección de medialización con un material temporal tienen menor probabilidad de requerir un procedimiento definitivo que los pacientes que inicialmente fueron manejados con observación o terapia fonoaudiológica ${ }^{10-13}$.

De los materiales de inyección temporales, el ácido hialurónico se ha convertido en uno de los más utilizados. Considerando que el ácido hialurónico es uno de los componentes naturales del espacio de Reinke, entre sus ventajas se contarían que su inyección no induce una respuesta inflamatoria local, que mantiene la viscoelasticidad de la lámina propia superficial, y que se mantiene estable después de la inyección. Además se cree que el ácido hialurónico inyectado tiene la capacidad de estimular el crecimiento de nuevo tejido conectivo, atrayendo a los fibroblastos, generando nuevo colágeno y macromoléculas de la matriz extracelular, de esta forma proporcionaría llenado a largo plazo y la elasticidad de la cuerda vocal ${ }^{14}$. Existen dos productos comercialmente disponibles de ácido hialurónico: Hylaform ${ }^{\circledR}$ (fabricado a partir de crestas de gallo) y Restylane ${ }^{\circledR}$ (producido por técnicas de ingeniería microbiológica). En relación a este último, se ha informado que tendría una duración de 4 a 6 meses. Además, los estudios histológicos en modelos animales han mostrado evidencia microscópica mínima de estas sustancias a los 6 meses de la inyección ${ }^{15-17}$.

En relación al abordaje, éste puede ser transoral, transtirohioideo, transtiroideo o transcricotiroide $0^{5}$.

\section{OBJETIVO}

Revisar la experiencia pionera del Hospital Clínico de la Pontificia Universidad Católica de Chile en la inyección de medialización de cuerda vocal con ácido hialurónico (Restylane ${ }^{\circledR}$ ) evaluando su efectividad en la mejoría de la voz, tolerancia al procedimiento y tasa de complicaciones.

\section{MATERIAL Y MÉTODO}

Se realizó un estudio de tipo retrospectivo en el Servicio de Otorrinolaringología del Hospital Clínico de la Universidad Católica de Chile. Se revisaron los registros de la Unidad de Voz del Departamento de Otorrinolaringología entre junio de 2012 y agosto de 2014, identificándose los pacientes con diagnóstico de insuficiencia glótica con indicación de inyección de medialización de cuerda vocal con ácido hialurónico (Restylane ${ }^{\circledR}$ ), ingresando al estudio los pacientes a los que se les realizó el procedimiento.

Se recolectaron los datos de edad, sexo, diagnóstico, lateralidad, abordaje, puntaje de la encuesta de calidad de vida (Voice Related Quality of Life, VRQOL) $)^{18}$ pre y posprocedimiento, número de intentos de inyección y complicaciones reportadas.

Las variables se describen a través de promedios o frecuencias porcentuales.

\section{RESULTADOS}

Durante el período de estudio se indicó la inyección de medialización de cuerda vocal con Restylane ${ }^{\circledR}$ a 36 pacientes con insuficiencia glótica, realizándose el procedimiento a 15 de ellos $(41,6 \%)$.

El promedio de edad de los pacientes sometidos al procedimiento es de 52,7 años con un rango entre 28 y 85 años. De ellos, el $47 \%$ corresponde a mujeres y el $53 \%$ a hombres.

La causa más frecuente de insuficiencia glótica fue la parálisis cordal unilateral en el $73 \%$ de los casos (11 pacientes), seguido por la paresia cordal unilateral en $13 \%$ (2 pacientes), y finalmente el sulcus vocalis y la presbilaringe con $7 \%$ cada uno. Las causas de las parálisis cordales fueron: posquirúrgica en 7 pacientes, secundaria a enfermedad neurológica en 2 (accidente vascular encefálico y síndrome de Wallenberg), secundario a compresión tumoral en uno (metástasis cervical de tumor testicular) e idiopático en 1 caso (Figura 1). Las parálisis 


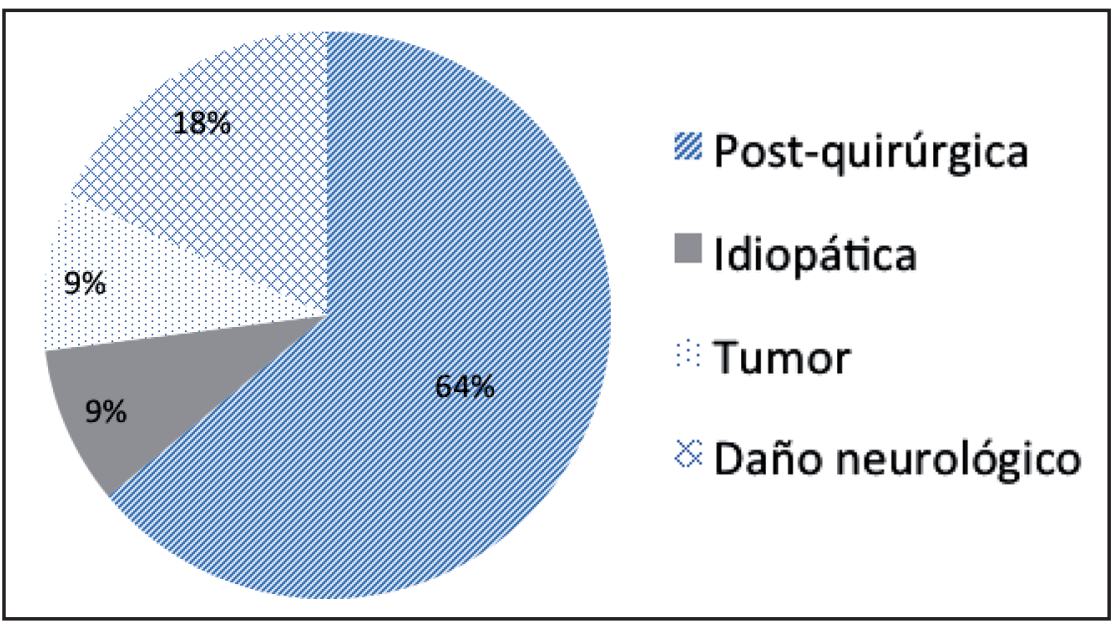

Figura 1. Causas de parálisis cordal unilateral.

cordales fueron izquierdas en el $73 \%$ de los casos, mientras que la cuerda vocal afectada en ambos pacientes con paresia cordal fue la derecha.

Todos los procedimientos fueron realizados en pacientes despiertos, por dos otorrinolaringólogos expertos. Se utilizó la técnica de inyección percutánea en todos los casos, con abordaje transtirohioideo en el $60 \%$ de los pacientes y transtiroideo en el $40 \%$ restante (todas pacientes de sexo femenino). La cantidad total de material inyectado varió de acuerdo al grado de insuficiencia glótica y se tituló durante el procedimiento en relación al resultado vocal del paciente, siendo aproximadamente de 0,5 $\mathrm{ml}$ por cuerda vocal.
La inyección en los pacientes con parálisis y paresia cordal fue unilateral. En ambos pacientes con presbilaringe y sulcus vocalis la inyección fue bilateral.

En 14 de los 15 pacientes la inyección se realizó en un tiempo, y sólo en un paciente se requirieron 2 sesiones para completar el procedimiento.

Durante el procedimiento no se produjo ningún incidente, así como tampoco se reportó ninguna complicación posterior.

La encuesta de calidad de vida VRQOL tuvo un promedio preprocedimiento de $51,4 \%$ (SD 22,4) y posprocedimiento de $88,6 \%$ (SD 10,6) (Figura 2). El $100 \%$ de los pacientes mostró cambios significa-

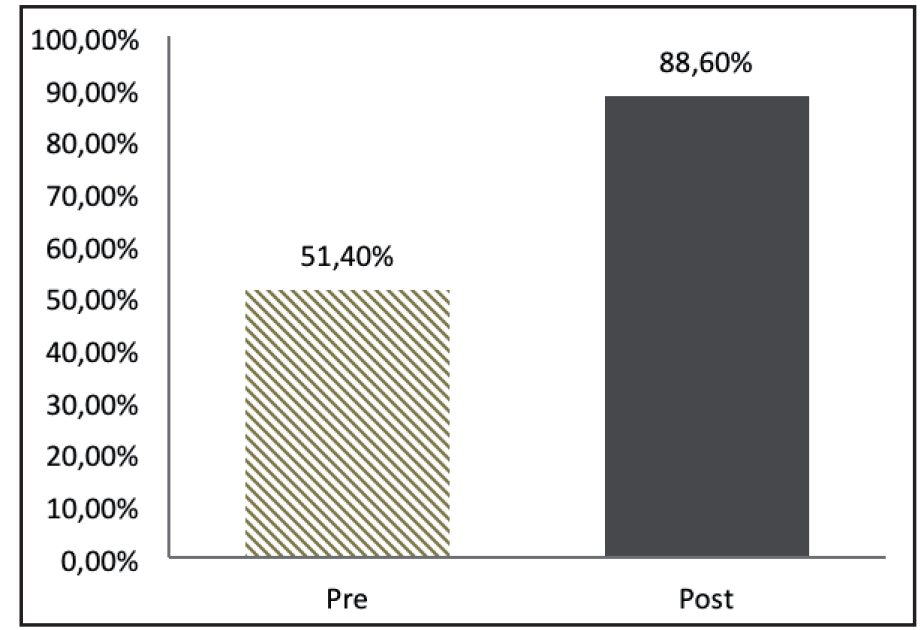

Figura 2. Resultado de la encuesta VRQOL pre y posinyección de medialización de cuerdas vocales. 
tivos en relación a esta medición con un promedio de cambio de $37 \%$.

\section{DISCUSIÓN}

La inyección de medialización de cuerda vocal con materiales temporales, como el ácido hialurónico, ha ido ganando terreno en el manejo de la insuficiencia glótica. Ésta se puede realizar vía laringoscopía directa bajo anestesia general 0 vía percutánea en la consulta con el paciente despierto. De estas dos opciones, la alternativa con paciente vigil ha logrado imponerse gracias a varias ventajas. Dentro de ellas destaca la posibilidad de ir "afinando" el resultado vocal durante el procedimiento, ya que se puede evaluar en tiempo real la calidad de la voz, guiando de este modo el volumen ideal de material inyectado. Además entrega mayor comodidad al paciente ya que no requiere hospitalización, redundando en un menor costo. Por otro lado, los resultados son rápidos, existe un mínimo tiempo de inactividad y los resultados serían comparables a los obtenidos en procedimientos realizados bajo anestesia general ${ }^{5}$. En este sentido, Johns comparó los VRQOL pre y posinyección de pacientes a quienes se les realizó una laringoplastía de inyección con anestesia general versus anestesia local, encontrando resultados vocales comparables ${ }^{19}$.

Si bien existe una creencia ampliamente aceptada de que la laringoplastía de inyección con materiales temporales sólo ofrece beneficios a corto plazo, estudios recientes han demostrado que esta intervención puede proporcionar una mejoría que se prolonga más allá del período de reabsorción, disminuyendo la probabilidad de una laringoplastía de medialización permanente (tiroplastía tipo I) en comparación con los pacientes que inicialmente optaron por observación o terapia fonoaudiológi$\mathrm{Ca}^{10}$. Los primeros en demostrarlo fueron Zeitels ${ }^{13}$ y Klein ${ }^{12}$ en 2010, resultados que han sido corroborados posteriormente por Courey ${ }^{10}$ en 2011 y 2012, entre otros. Klein ${ }^{12}$ evidenció que la mayoría de los pacientes con parálisis cordal unilateral potencialmente recuperable sometidos a una laringoplastía de medialización temporal no requieren una medialización quirúrgica permanente. Por otro lado, Zeitels ${ }^{13}$ concluyó que los pacientes que reciben una inyección de medialización temporal precozmente (dentro de los primeros 6 meses desde el inicio de la parálisis) tenían menor probabilidad de requerir una reconstrucción transcervical, postulando que esto se debería a que la inyección temprana sitúa la cuerda vocal en una posición más favorable para la fonación, posición que es luego mantenida por la reinervación sinquinética, en contraste con la posición final de una cuerda vocal lateralizada determinada sólo por la reinervación.

Por su parte, Courey en $2011^{10}$ observó que los pacientes sometidos a una inyección de medialización de cuerda vocal con un agente temporal para tratar la insuficiencia glótica tuvieron significativamente menos probabilidades de someterse a una laringoplastía de medialización permanente en comparación con aquellos pacientes que fueron tratados sólo con tratamiento conservador con $26,3 \%$ vs $65,7 \%$ respectivamente $(p=0,0131)$. Además se observó que la mayoría de los parámetros de CAPE-V (voz soplada, tono y proyección vocal) fueron significativamente mejores en los pacientes tratados con inyección de medialización temporal, así como también la mayoría de los parámetros laringoscópicos (insuficiencia glótica, arqueo de cuerda vocal y contacto de apófisis vocales ${ }^{11}$.

Por otra parte, este tipo de procedimiento es bastante seguro, sin embargo, se describen algunas complicaciones: en primer lugar, tenemos la mala tolerancia del paciente (dolor, ansiedad 0 tos), mala visualización de la laringe y las reacciones vasovagales. Estas complicaciones podrían determinar la necesidad de suspender el procedimiento. En segundo lugar, tenemos las complicaciones relacionadas al procedimiento propiamente tal, donde podemos mencionar las hemorragias cordales, inyección superficial del material (lámina propia superficial), rápida absorción del material, obstrucción de la vía aérea por un hematoma cervical o por un laringoespasmo, y reacciones adversas al material de relleno. En la literatura se describe una tasa de $5,7 \%$ de procedimientos que se tienen que suspender; $1,2 \%$ de hemorragias cordales, $5 \%$ de inyecciones de material de relleno mal situadas; $3,6 \%$ de rápida absorción del material inyectado, requiriendo una segunda laringoplastía ${ }^{19}$, así como también está publicada la ocurrencia muy poco habitual de reacciones adver- 
sas serias al ácido hialurónico ${ }^{20}$. En nuestro estudio, sólo en una paciente $(7 \%)$ se debió realizar la inyección en dos tiempos debido a mala tolerancia inicial y no se observó ninguna complicación relacionada al procedimiento.

Con respecto a la etiología de la insuficiencia glótica, los hallazgos de nuestro estudio son similares a lo encontrado en la literatura, donde la mayoría de las insuficiencias glóticas son producidas por parálisis cordal uni o bilaterales secundarias a traumas producidos en cirugías como por ejemplo la tiroidectomía ${ }^{21}$.

\section{CONCLUSIÓN}

La inyección de medialización temporal de cuerda vocal es un procedimiento bien tolerado, seguro y sencillo, que permite mejoras significativas inmediatas en la calidad vocal de los pacientes.

Además, el ácido hialurónico es un material temporal seguro y eficaz para la inyección de medialización de cuerda vocal.

\section{BIBLIOGRAFÍA}

1. TUCKER HM. Vocal cord paralysis-etiology and management. Laryngoscope 1980; 90: 58590.

2. Sulica L, Rosen CA, Postma GN, et al. Current practice in injection augmentation of the vocal folds: indications, treatment principles, techniques, and complications. Laryngoscope 2010; 120: 319-25.

3. BRUENINGS W. Uebereineneue Behandlus methode der Rekurrenslaehmung. Verhandl Der Dtsch Laryngol 1911; 17: 93.

4. Ward PH, Hanson DG, Abemayor E. Transcutaneous teflon injection of the paralyzed vocal cord: a new technique. Laryngoscope 1985; 95: 644-9.

5. Rosen, Clark A et al. Advances in office based diagnosis and treatment in laryngology. The Laryngoscope 2009; 119.S2: S185-S212.

6. Clary, Matthew S, Benjamin M Milam, and Mark $S$ COUREY. Office based vocal fold injection with the laryngeal introduce technique. The Laryngoscope 2014.
7. MODI, VIKASH K. Vocal fold injection medialization laryngoplasty. Advances in Oto Rhino Laryngology 2012; 73: 90.

8. Verma, Sunil P, and Seth h Dalley. Office based injection laryngoplasty for the management of unilateral vocal fold paralysis. Journal of Voice 2014; 28(3): 382-6.

9. KWONA TK, Buckmireb R. Injection laryngoplasty for management of unilateral vocal fold paralysis. Curr Opin Otolaryngol Head Neck Surg 2004; 12 : 538-42.

10. Yung KC, Likhterov I, Courey MS. Effect of temporary vocal fold injection medialization on the rate of permanent medialization laryngoplasty in unilateral vocal fold paralysis patients. Laryngoscope 2011; 121: 2191-4.

11. Prendes BL, Yung KC, Likhterov I, Schneider SL, Al-Jurf SA, Courey MS. Long-term effects of injection laryngoplasty with a temporary agent on voice quality and vocal fold position. Laryngoscope 2012; 122 : 2227-33.

12. Arviso LC, Johns MM, Mathison CC, Klein AM. Long-Term Outcomes of Injection Laryngoplasty in Patients With Potentially Recoverable Vocal Fold Paralysis. Laryngoscope 2010; 120: 223740.

13. Friedman Ad, Burns JA, Heaton JT, Zeitels SM. Early versus late injection medialization for unilateral vocal cord paralysis. Laryngoscope 2010; 120: 2042-6.

14. Kimura M, Nito T, Sakakibara KI et al. Clinical experience with collagen injection of the vocal fold: A study of 155 patients. Auris Nasus Larynx 2008; 35: 67-75.

15. Hertegard S, Hallen L, Laurent C, Lindstrom E, Olofsson K, Testad P, et Al. Cross-linked hyaluronan versus collagen for injection treatment of glottal insufficiency: 2-year follow-up. Acta Otolaryngol 2004; 124: 1208-14.

16. Moltenı G, Bergaminı G, Ricci-Maccarinı A, Marchese C, Ghidini A, Alicandri-Ciufelli M, et AL. Auto-cross linked hyaluronan gel injections in phonosurgery. Otolaryngol Head Neck Surg 2010; 142(4): 547-53.

17. Rudolf, Reiter, and Brosch Sibylle. Laringoplasty with hyaluronic acid in patients with unilateral vocal fold paralysis. Journal of Voice 2012; 26(6): 785-91. 
18. Hogikyan, Norman D and Girish Sethuraman. Validation of an instrument to measure voice-related quality of life (V-RQOL). Journal of Voice 1999; 13(4): 557-69.

19. Mathison CC, Villari CR, Klein AM, Johns MM. Comparison of Outcomes and Complications between Awake and Asleep Injection Laryngoplasty: A CaseControl Study. Laryngoscope 2009; 119: 1417-23.
20. Shamanna SG, Bosch JD. Injection laryngoplasty: a serious reaction to hialuronic acid. J Otolaryngol Head Neck Surg 2011; (40)5: E39-42.

21. Bothe Carolina et al. Aetiology and treatment of vocal fold paralysis: Retrospective study of 108 patients. Acta Otorrinolaringológica 2014; 65(4): 225-30.

E mail: pbadia@med.puc.cl 